\title{
Efficacy of Balloon-Guiding Catheter for Mechanical Thrombectomy in Patients with Anterior Circulation Ischemic Stroke
}

\author{
Jae-Sang Oh, M.D., Seok-Mann Yoon, M.D., Ph.D., Jai-Joon Shim, M.D., Ph.D., Jae-Won Doh, M.D., Ph.D., \\ Hack-Gun Bae, M.D., Ph.D., Kyeong-Seok Lee, M.D., Ph.D. \\ Department of Neurosurgery, Soonchunhyang University Cheonan Hospital, Cheonan, Korea
}

Objective : To evaluate the efficacy of balloon guiding catheter (BGC) during thrombectomy in anterior circulation ischemic stroke. Methods : Sixty-two patients with acute anterior circulation ischemic stroke were treated with thrombectomy using a Solitaire stent from 2011 to 2016. Patients were divided into the BGC group $(n=24,39 \%)$ and the non-BGC group $(n=38,61 \%)$. The number of retrievals, procedure time, thrombolysis in cerebral infarction (TICI) grade, presence of distal emboli, and clinical outcomes at 3 months were evaluated.

Results : Successful recanalization was more frequent in BGC than in non-BGC ( $83 \%$ vs. $66 \%, p=0.13$ ). Distal emboli occurred less in BGC than in non-BGC (23.1\% vs. $57.1 \%, p=0.02)$. Good clinical outcome was more frequent in BGC than in non-BGC ( $50 \%$ vs. $16 \%$, $p=0.03$ ). The multivariate analysis showed that use of BGC was the only independent predictor of good clinical outcome (odds ratio, 5.19 : 95\% confidence interval, 1.07-25.11). More patients in BGC were successfully recanalized in internal carotid artery (ICA) occlusion with small retrieval numbers ( $<3)$ than those in non-BGC ( $70 \%$ vs. $24 \%, p=0.005)$. In successfully recanalized ICA occlusion, distal emboli did not occur in BGC, whereas nine patients had distal emboli in non-BGC ( $0 \%$ vs. $75 \%, p=0.001)$ and good clinical outcome was superior in BGC than in non-BGC (55.6\% vs. 8.3\%, $p=0.01)$.

Conclusion : A BGC significantly reduces the number of retrievals and the occurrence of distal emboli, thereby resulting in better clinical outcomes in patients with anterior circulation ischemic stroke, particularly with ICA occlusion.

Key Words : Balloon guiding catheter · Anterior circulation · Ischemic stroke $\cdot$ Thrombectomy $\cdot$ ICA occlusion.

\section{INTRODUCTION}

An intra-arterial (IA) mechanical thrombectomy device should be simple, convenient, and not create procedural-related complications. Multiple retrieval attempts during IA thrombectomy can increase the risk of procedural-related complications (vessel dissection or perforation) and downstream emboli. Procedural release of emboli may be a factor in poor outcome and a modifiable risk. The time and effort of the endovascular neurosurgeon should be used to achieve successful recanalization with few complications. Considering these points, the balloon-guiding catheter (BGC) is useful to

- Received : September 26, 2016 •Revised : November 22, 2016 •Accepted : December 9, 2016

- Address for reprints : Seok-Mann Yoon, M.D., Ph.D.

Department of Neurosurgery, Soonchunhyang University Cheonan Hospital, 31 Soonchunhyang 6-gil, Dongnam-gu, Cheonan 31151, Korea Tel : +82-41-570-3649, Fax : +82-41-572-9297, E-mail : smyoonns@gmail.com

This is an Open Access article distributed under the terms of the Creative Commons Attribution Non-Commercial License (http://creativecommons.org/licenses/by-nc/4.0) which permits unrestricted non-commercial use, distribution, and reproduction in any medium, provided the original work is properly cited. 
reduce procedure time and effort, as well as to obtain successful recanalization during IA thrombectomy ${ }^{10)}$. A BGC can also reduce iatrogenic emboli and it may be related with good outcome $^{2)}$. This study was conducted to evaluate the efficacy and usefulness of a BGC during IA mechanical thrombectomy in patients with an anterior circulation ischemic stroke.

\section{MATERIALS AND METHODS}

We retrospectively evaluated 62 patients who were treated with IA mechanical thrombectomy for an acute anterior circulation ischemic stroke from January 2011 to January 2016. The Solitaire FR (Medtronic Neurovascular, Irvine, CA, USA) was used as the first-line device, and Cello (Medtronic Neurovascular) was used for balloon guiding. And we did not use any other distal access catheter or retrieval devices. The tip of balloon was positioned on proximal internal carotid artery (ICA) (within $3 \mathrm{~cm}$ above CCA bifurcation). And eligible patients were randomized to receive the mechanical thrombectomy with Solitaire FR and BGC or Solitaire thrombectomy alone, according to surgeon's preference. Patients were divided into the BGC $(n=24,39 \%)$ and non-BGC groups $(n=38,61 \%)$. Eligible patients were : age $\geq 18$ years, $8 \leq$ NIHSS $\leq 30$, within 8 hours after symptom onset, platelet count $>80,000$, ASPECT $\geq 6$ and INR $<2.5$.

Baseline and post-procedural brain and angiographic images were reviewed by two endovascular neurosurgeons. Pretreatment Alberta Stroke Program Early CT Score (ASPECTS) was analyzed in 61 patients using computed tomography (CT) or angio-CT, except in one patient. Arterial patency on pre and post-procedural angiograms was classified using the modified Thrombolysis in Cerebral Infarction (TICI) score ${ }^{13)}$. Post-procedural intracranial hemorrhage was defined as any parenchymal hemorrhage, subarachnoid hemorrhage, or intraventricular hemorrhage. Of these, symptomatic intracranial hemorrhage was defined when neurological condition deteriorated (National Institutes of Health Stroke Scale [NIHSS] score $\geq 4$ within 24 hours) or additional surgical intervention (decompressive craniectomy or extraventricular drainage) was needed $^{12)}$. Clinical and radiological variables, such as the capillary index score (CIS), puncture time from symptom onset, procedure time, number of retrievals, the presence of distal emboli, TICI grade, and clinical outcome were evaluated ret- rospectively. Successful recanalization was defined when TICI $2 \mathrm{~b}$ or 3 on post IA thrombectomy angiography was achieved. Number of retrievals was defined as the number of Solitaire stent retrievals under deployment over the thrombus. Two groups were formed for the statistical analysis, such as $<3$ (small retrieval numbers) and $\geq 3$ retrievals. One time retrieval with TICI 3 was defined when total recanalization (TICI 3) was obtained on the first attempt. Good clinical outcome was defined as a modified Rankin score (mRS) $\leq 2$ at 3 months.

\section{Procedure time}

Routine angiography is not performed in our center on the non-ischemic side of the anterior or posterior circulation before IA mechanical thrombectomy. Therefore, procedure time excluded the time taken for routine angiography on the nonischemic side of the anterior or posterior circulation. Procedure time was divided as follows : 1) total procedure time : time taken from femoral puncture to finishing the procedure, 2) guiding time : time from the femoral puncture to position the guiding catheter (or BCG) on proximal ICA, 3) thrombectomy time : time to perform the mechanical thrombectomy to recanalization of the occluded vessel or end of the procedure after positioning the guiding catheter.

\section{Distal emboli}

Distal emboli were defined as visible thrombus fragments or emboli downstream from the primary site of occlusion to the distal segment or new other territory on angiography during IA mechanical thrombectomy. Distal emboli sometimes occurred on ipsilateral M2, distal middle cerebral artery (MCA) branch or anterior cerebral artery (ACA) territory after several Solitaire stent retrievals in cases of M1 occlusion. Distal emboli occurred on ipsilateral A2, M2, distal ACA, or the MCA branch in cases of carotid T occlusion.

\section{Procedure protocol}

A $5 \mathrm{~F}$ diagnostic catheter with $8 \mathrm{~F}$ shuttle were introduced into the common carotid artery (CCA) using a coaxial technique, and a CCA angiogram was obtained. After the tip of balloon was positioned on proximal ICA (within $3 \mathrm{~cm}$ above CCA bifurcation), the Solitaire FR device was delivered and deployed over the thrombus through 018 or 027 microcatheter while the microcatheter covered the proximal marker of the 
stent to prevent unexpected stent detachment during retrieval. A control angiogram was performed to determine the immediate reperfusion status. The stent was left deployed for $5 \mathrm{~min}$. Then, the BCG was inflated to provide proximal ICA occlusion and flow arrest during retrieval. The Solitaire stent and microcatheter were drawn slowly under constant aspiration with a 50-mL syringe through the BGC. After the stent and microcatheter were removed, constant aspiration was maintained until thrombi were not detected in aspirated blood.

\section{Statistical analysis}

Quantitative variables were expressed as mean \pm standard deviation. Baseline characteristics were compared between the BGC and non-BGC groups using Fisher exact or $\chi^{2}$ analysis for categorical variables. Continuous variables were analyzed with Student's t-test or Mann-Whitney U test. For evaluation of the independent predictors of good clinical outcome of IA Solitaire thrombectomy for acute ischemic stroke, variables with $p$-values $<0.10$ were included in multivariate binary logistic regression model. $p$-value of $<0.05$ was considered significant. Odds ratios (ORs) and 95\% confidence intervals (CIs) were determined for the logistic regression analysis. SPSS ver. 18 software (IBM Corp., Armonk, NY, USA) was used for all statistical analysis.

Table 1. Univariate comparison of the BGC and non-BGC groups

\begin{tabular}{|c|c|c|c|c|}
\hline Variable & $B G C(n=24)$ & Non-BGC $(n=38)$ & Total $(n=62)$ & $p$-value \\
\hline Age, y, mean (SD) & $65.1(9)$ & $63.8(14)$ & $64.3(12)$ & 0.7 \\
\hline Women $(n, \%)$ & $12(50)$ & $11(28.9)$ & 23 (37.1) & $0.09^{*}$ \\
\hline \multicolumn{5}{|l|}{ Past history $(n, \%)$} \\
\hline Hypertension & $11(45.8)$ & $12(34.2)$ & $24(38.7)$ & 0.36 \\
\hline Atrial fibrillation & $1(4.2)$ & $3(7.9)$ & $4(6.5)$ & 0.56 \\
\hline Diabetes mellitus & $4(16.7)$ & $5(13.2)$ & $9(14.5)$ & 0.7 \\
\hline Hyperlipidemia & $1(4.2)$ & $3(7.9)$ & $4(6.5)$ & 0.56 \\
\hline Antiplatelet & $2(8.3)$ & $2(5.2)$ & $4(6.5)$ & 0.63 \\
\hline Anticoagulant & $0(0)$ & $2(5.3)$ & $2(3.2)$ & 0.25 \\
\hline \multicolumn{5}{|l|}{ Laboratory finding (mean, SD) } \\
\hline Hemoglobin (g/dL) & $13.8(11-17)$ & $13.9(10-16)$ & $13.9(1.7)$ & 0.91 \\
\hline Platelet count $\left(x 10^{3} / \mu \ell\right)$ & $225(86-372)$ & $213(136-335)$ & $218(60)$ & 0.45 \\
\hline Prothrombin time (INR) & $1.06(0.87-1.32)$ & $1.09(0.92-2.49)$ & $1.08(0.23)$ & 0.64 \\
\hline Mean initial NIHSS score (mean, SD) & $15.5(5.7)$ & $15.8(5.5)$ & $15.7(5.5)$ & 0.83 \\
\hline Location of thrombus (n, \%) & & & & 0.74 \\
\hline ICA & $11(45.8)$ & $19(50)$ & $30(48.4)$ & \\
\hline MCA & $13(54.2)$ & $19(50)$ & $32(51.6)$ & \\
\hline \multicolumn{5}{|l|}{ Side $(n, \%)$} \\
\hline Left & $10(41.7)$ & $26(68.4)$ & $36(58.1)$ & $0.03^{*}$ \\
\hline Right & $14(58.3)$ & $12(31.6)$ & $26(41.9)$ & \\
\hline IV tPA (n, \%) & $12(50)$ & $15(39.5)$ & $27(43.5)$ & 0.41 \\
\hline ASPECTS $(n, \%)$ & & & & 0.99 \\
\hline 6 & $3(12.5)$ & $5(13.2)$ & $8(12.9)$ & \\
\hline$>6$ & $20(87.0)$ & $33(86.8)$ & $53(86.9)$ & \\
\hline $\operatorname{CIS}(n, \%)$ & & & & 0.78 \\
\hline $\operatorname{Bad}(0,1)$ & $6(26.1)$ & $10(29.4)$ & $16(28.1)$ & \\
\hline Good $(2,3)$ & $17(73.6)$ & $24(70.6)$ & $41(71.9)$ & \\
\hline
\end{tabular}

${ }^{*} p<0.05$ as significant. SD : standard deviation, BGC : balloon-guiding catheter, NIHSS : National Institutes of Health Stoke Scale, ICA : internal carotid artery, MCA : middle cerebral artery, tPA : tissue-plasminogen activator, IA : intra-arterial, ASPECTS : Alberta Stroke Program Early CT Score, CIS : capillary index score 


\section{RESULTS}

Of these 83 patients, 62 (74.6\%) underwent IA mechanical thrombectomy for an anterior circulation ischemic stroke. Twenty-four of the $62(38.7 \%)$ patients had a BGC placed for IA mechanical thrombectomy due to an anterior circulation ischemic stroke. Excluded patients had only received an aspiration thrombectomy or other mechanical device without a Solitaire FR stent.

Baseline clinical and neuroimaging characteristics of the patients are shown in Table 1. The median NIHSS score on admission was 15 (range, 2-32). Twenty-seven (43.5\%) patients were treated with IV tPA (full dose) before IA mechanical thrombectomy and the others had contraindications for IV
tPA. Baseline ASPECTS on the CT examination was checked in 61 of $62(98.4 \%)$ patients. One patient had a MCA occlusion during proximal carotid artery stenting, so direct IA mechanical thrombectomy was performed without CT or magnetic resonance imaging (MRI). The CIS was readable in 57 of 62 (91.9\%) patients with an anterior circulation lesion on preprocedural brain angio-CT. No differences were observed in the baseline or neuroimaging characteristics between the BGC and non-BGC groups. However, left side occlusion was significantly more common in the non-BGC than that in the BGC group (68.4\% vs. $41.7 \%, p=0.03)$.

The procedural and clinical results between the $\mathrm{BGC}$ and non-BGC groups are summarized in Table 2. Femoral puncture time from symptom onset was not different between the

Table 2. Radiographic and clinical outcomes of IA mechanical thrombectomy in patients with an anterior circulation ischemic stroke

\begin{tabular}{|c|c|c|c|c|}
\hline Variable & $B G C(n=24)$ & Non-BGC $(n=38)$ & Total $(n=62)$ & $p$-value \\
\hline $\begin{array}{l}\text { Mean puncture time from symptom onset } \\
\text { (SD, median, range, min) }\end{array}$ & $199(80,184,30-485)$ & $238(89,247,30-480)$ & $223(87,205,30-485)$ & 0.089 \\
\hline \multicolumn{5}{|l|}{ Times of (mean, SD, min) } \\
\hline Total procedure time & $78.8(33)$ & $92.7(45.6)$ & $87(41)$ & 0.46 \\
\hline Guiding time & $20.3(13.7)$ & $18.3(18)$ & $19(16)$ & 0.40 \\
\hline Thrombectomy time & $58.5(28.8)$ & $74.4(45.6)$ & $68(40)$ & 0.31 \\
\hline Mean number of retrievals & $2.4(1.3)$ & $3.7(1.7)$ & $3.1(1.6)$ & $0.004^{*}$ \\
\hline \multicolumn{5}{|l|}{ Number of retrievals ( $n, \%)$} \\
\hline$<3$ & $16(66.7)$ & 7 (18.4) & $23(37.1)$ & $<0.001^{*}$ \\
\hline$\geq 3$ & $8(33.3)$ & $31(81.6)$ & $39(62.9)$ & \\
\hline One time retrieval with $\mathrm{TICl} 3(\mathrm{n}, \%)$ & $8(33.3)$ & $3(7.9)$ & $11(17.7)$ & $0.01^{*}$ \\
\hline Distal emboli $(n, \%)$ & $6(23.1)$ & $20(57.1)$ & $26(44.8)$ & $0.02^{*}$ \\
\hline \multicolumn{5}{|l|}{$\mathrm{TICl}$ grade $(\mathrm{n}, \%)$} \\
\hline $0 \& 1 \& 2 a$ & $4(16.7)$ & $13(34.2)$ & $17(27.4)$ & 0.13 \\
\hline $2 \mathrm{~b} \& 3$ & $20(83.3)$ & $25(65.8)$ & $45(72.6)$ & \\
\hline 3 & $16(66.7)$ & $17(44.7)$ & $33(53.2)$ & 0.092 \\
\hline \multicolumn{5}{|l|}{ Postop Image findings (n, \%) } \\
\hline Dye leakage & $13(54.2)$ & $34(89.5)$ & $47(75.8)$ & $0.002^{*}$ \\
\hline $\mathrm{ICH}$ & $7(29.2)$ & $5(13.2)$ & $12(19.4)$ & 0.12 \\
\hline Symptomatic ICH & $4(16.7)$ & $4(10.5)$ & $8(12.9)$ & 0.48 \\
\hline \multicolumn{5}{|l|}{ Clinical outcomes at 3 months (n, \%) } \\
\hline Good (mRS $\leq 2)$ & $12(50)$ & $6(15.8)$ & $18(29)$ & $0.004^{*}$ \\
\hline Poor $(m R S>2)$ & $12(50)$ & $32(84.2)$ & $44(71)$ & \\
\hline Mortality (n, \%) & $2(8.3)$ & $6(15.8)$ & 8 (12.9) & 0.39 \\
\hline
\end{tabular}

${ }^{*} p<0.05$ as significant. IA : intra-arterial, BGC : balloon-guiding catheter, SD : standard deviation, TICI : Thrombolysis in Cerebral Infarction, ICH : intracranial hemorrhage, $\mathrm{mRS}$ : modified Rankin scale 
groups (BGC vs. non-BGC : 199 vs. $238 \mathrm{~min}, p=0.08$ ). Distal emboli on angiography were less frequent in the BGC than those in the non-BGC group (23.1\% vs. $57.1 \%$, 6 vs. 20 , $p=0.02)$. Successful recanalization $(\geq 2 b)$ and total recanalized TICI grade 3 reperfusion were more frequent in the $\mathrm{BGC}$ than those in the non-BGC group ( $p>0.05)$. The overall technical success rate to deploy the Solitaire stent on the occlusion site was $100 \%$, and no technical complications were encountered. Post-procedural dye-leakage was significantly less frequent in the BGC than that in the non-BGC group $(p=0.00)$. Good clinical outcomes $(\mathrm{mRS} \leq 2)$ at 3 months were more frequent in the in BGC than those in the non-BCG group (50\% vs. $15.8 \%, 12$ vs. $6, p=0.00$ ). Thrombectomy time were more shorter in the BGC than those in the non-BGC group $(p>0.05)$. Significantly fewer mean retrievals were required in the successfully recanalized TICI $(\geq 2 \mathrm{~b})$ group than that in the poorly recanalized TICI group $(\leq 2 \mathrm{a})(2.7 \pm 1.5$ vs. $4.0 \pm 1.7$, $p=0.00)$. And small retrieval numbers were more frequent in BGC group ( $66.7 \%$ vs. $18.4 \%, 16$ vs. $7, p=0.00)$. One time retrieval with TICI 3 was more frequent in the BGC than that in the non-BGC group (33.3\% vs. $7.9 \%, 8$ vs. $3, p=0.01$ )

\section{Subgroup analysis}

\section{Successful recanalization group (Table 3)}

Using the BGC reduced the number of retrievals for successful recanalization. Fewer retrievals were required in the $\mathrm{BGC}$ than that in the non-BGC group $(2.2 \pm 1.4$ vs. $3.2 \pm 1.5$, $p=0.03$ ). Significantly more patients in the BGC group were successfully recanalized with small retrieval numbers than those in the non-BGC group (70\% vs. $24 \%, 14$ vs. $6, p=0.00$ ). One time retrieval with TICI 3 was also more frequent in the BGC than that in the non-BGC group (40\% vs. $12 \%, 8$ vs. 3 , $p=0.03$ ). Distal emboli occurred less frequently in the BGC than those in the non-BGC group (25\% vs. $56 \%, 5$ vs. 14 , $p=0.03$ ). Good clinical outcome (mRS $\leq 2)$ at 3 months were more frequent in the BGC compared with that in the nonBCG group (55\% vs. $13 \%, 11$ vs. $3, p=0.00)$.

\section{Successful recanalization in the ICA occlusion (Table 4)}

Significantly more patients with an ICA occlusion in the BGC group were successfully recanalized with small retrieval numbers than those in the non-BGC group (77.8\% vs. $16.7 \%$, 7 vs. $2, p=0.00)$. One time retrieval with TICI 3 was also more frequent in the BGC than that in the non-BGC group (44.4\% vs. $8.3 \%, 4$ vs. $1, p=0.05$ ). No distal emboli were detected this subgroup of the BGC group, but nine patients had distal emboli in the non-BGC group ( $0 \%$ vs. $75 \%, p=0.00)$. Good clinical outcomes (mRS $\leq 2)$ at 3 months were more frequent in the BGC compared with those in the non-BCG (55.6\% vs. $8.3 \%, 5$ vs. $1, p=0.01)$.

\section{Successful recanalization in the MCA occlusion (Table 5)}

Left-sided occlusion was less frequent in the BGC than that in the non-BGC group (38.5\% vs. $73.7 \%, 5$ vs. $14, p=0.04$ ). Overall successful recanalization for MCA occlusion was achieved in 24 (75\%) patients. Small retrieval numbers with successful recanalization on MCA occlusion was higher in BGC than non-BGC group (63.6\% vs. $30.8 \%, 7$ vs. $4, p=0.10$ ) and one time retrieval with TICI 3 tended to be more frequent in the BGC than that in the non-BGC group (36.4\% vs. $15.4 \%$, 4 vs. $2, p=0.23$ ). The incidence of distal emboli on this subgroup had no difference between BGC group and non-BGC group (45.5\% vs. $38.5 \%, 5$ vs. $5, p>0.05$ ). Good clinical outcome at 3 months were more frequent in the BGC than those in the non-BGC group ( $54.5 \%$ vs. $15.4 \%, 6$ vs. 2 , $p=0.04$ ).

\section{Predictors of good clinical outcome}

Overall good clinical outcome (mRS $\leq 2)$ at 3 months was achieved in 29\% (18 of 62 patients). The multivariate analysis (adjusting for sex, right/left side, puncture time from symptom onset, total recanalized TICI 3, use of BGC, small retrieval numbers, and distal emboli) showed that use of a BGC was the only independent predictor of good clinical outcome (OR, 5.19; 95\% CI, 1.07-25.11, $p=0.04$; Table 6).

\section{DISCUSSION}

The North American Solitaire Acute Stroke Registry (NASA) study showed that a BGC improves revascularization, shortens procedure time, and improves clinical outcomes with the Solitaire stent ${ }^{10)}$. However, the NASA study did not demonstrate the actual time taken for IA thrombectomy except guiding time, and that study involved 10\% basilar artery occlusions and small proportion of ICA occlusions (22.7\%). In addition, a relatively long procedure time was reported. They did not divide procedure time into each steps such as time required to place guiding catheter, and actual thrombectomy 
Table 3. Univariate comparison between the BGC and non-BGC groups in patients who were successfully recanalized (TICl $2 b$ \& 3$)$

\begin{tabular}{|c|c|c|c|c|}
\hline Variable & $B G C(n=20)$ & Non-BGC $(n=25)$ & Total $(n=45)$ & $p$-value \\
\hline Age (SD, yrs) & $64(9)$ & $65(13)$ & $64(11)$ & 0.78 \\
\hline Women $(n, \%)$ & $9(45)$ & $7(28)$ & $16(35.6)$ & 0.23 \\
\hline Mean initial NIHSS score (mean, SD) & $16.1(6.1)$ & $14.4(5.6)$ & $15.2(5.8)$ & 0.35 \\
\hline \multicolumn{5}{|l|}{ Side $(n, \%)$} \\
\hline Left & $11(55)$ & $8(32)$ & $19(42.2)$ & 0.12 \\
\hline Right & $6(54.5)$ & $7(36.8)$ & $13(43.3)$ & \\
\hline IV tPA (n, \%) & $10(50)$ & $10(40)$ & $20(44.4)$ & 0.50 \\
\hline \multicolumn{5}{|l|}{ ASPECTS (n, \%) } \\
\hline 6 & $2(10.5)$ & $3(12)$ & $5(11.4)$ & 0.87 \\
\hline$>6$ & $17(89.5)$ & $22(88)$ & 39 (88.6) & \\
\hline \multicolumn{5}{|l|}{$\operatorname{CIS}(n, \%)$} \\
\hline $\operatorname{Bad}(0,1)$ & $4(20)$ & $8(36.4)$ & $12(28.6)$ & 0.24 \\
\hline Good $(2,3)$ & $16(80)$ & $14(63.6)$ & $30(71.4)$ & \\
\hline \multicolumn{5}{|l|}{ Location of thrombus (n, \%) } \\
\hline ICA & $11(55)$ & $13(52)$ & $24(53.3)$ & 0.84 \\
\hline MCA & $9(45)$ & $12(48)$ & $21(46.7)$ & \\
\hline Puncture time from symptom onset (mean, SD, min) & $199(84)$ & $222(79)$ & $212(81.9)$ & 0.34 \\
\hline \multicolumn{5}{|l|}{ Times of (mean, SD, min) } \\
\hline Total procedure time & $78(33)$ & $84(33)$ & $81(33)$ & 0.52 \\
\hline Guiding time & $21(14)$ & $18(17)$ & $19(17)$ & 0.56 \\
\hline Thrombectomy time & $57(29)$ & $66(34)$ & $62(32)$ & 0.33 \\
\hline Mean number of retrievals & $2.2(1.4)$ & $3.2(1.5)$ & $2.7(1.5)$ & $0.03^{*}$ \\
\hline \multicolumn{5}{|l|}{ Number of retrievals $(n, \%)$} \\
\hline$<3$ & $14(70)$ & $6(24)$ & $20(44.4)$ & $0.002^{*}$ \\
\hline$\geq 3$ & $6(30)$ & $19(79)$ & $25(55.6)$ & \\
\hline One time retrieval with $\mathrm{TICl} 3$ & $8(40)$ & $3(12)$ & $11(24.4)$ & $0.03^{*}$ \\
\hline Distal emboli (n, \%) & $5(25)$ & $14(56)$ & $19(42.2)$ & $0.036^{*}$ \\
\hline \multicolumn{5}{|l|}{ Good Clinical outcome (n, \%) } \\
\hline $\mathrm{mRS} \leq 2$ & $11(55)$ & $3(12)$ & $14(31)$ & $0.002^{*}$ \\
\hline
\end{tabular}

${ }^{*} p<0.05$ as significant. BGC : balloon guiding catheter, SD : standard deviation, TICl : Thrombolysis in Cerebral Infarction, mRS : modified Rankin scale, ICA : internal carotid artery, MCA : middle cerebral artery

time. And they did not investigate according to the successful recanalization or not. General anesthesia was performed in $58 \%$ of patients during IA mechanical thrombectomy in the NASA study. General anesthesia can produce a time-base bias and delay puncture time from onset, which is a very important prognostic factor in patients with an acute ischemic occlusion.

Our study tried to find the effect of BGC which could reduce the number of retrievals on successful recanalized group and the occurrence of distal emboli on ICA occlusion using subgroup analysis. All procedures were performed under the local anesthesia for prompt recanalization. To reduce the wasting time, we used pre-packed thrombectomy package which consisted of all materials (fabricated IV lines and hubs in advance, wire, gauze, etc.) to use in emergency thrombectomy. We divided total procedure time into three portions (total procedure time, guiding time, and thrombectomy time) to consider the time consumed in each steps, reflecting access 
Efficacy of Balloon Guiding Thrombectomy | Oh JS, et al.

Table 4. Univariate comparison between the BGC and non-BGC groups for patients with an ICA occlusion and a subgroup analysis (A) for those with a successful recanalization ( $\mathrm{TICl} 2 \mathrm{~b}$ or 3 )

\begin{tabular}{|c|c|c|c|c|}
\hline Variable & $B G C(n=11)$ & Non-BGC $(n=19)$ & Total $(n=30)$ & $p$-value \\
\hline Age (SD, yrs) & $65(8)$ & $65(14)$ & $65(12)$ & 0.85 \\
\hline Women $(n, \%)$ & $6(54.5)$ & $5(26.3)$ & $11(36.7)$ & 0.12 \\
\hline Mean initial NIHSS score (mean, SD) & $17.0(7.0)$ & $17.8(3.6)$ & $17.5(5.0)$ & 0.65 \\
\hline \multicolumn{5}{|l|}{ Side $(n, \%)$} \\
\hline Left & $5(45.5)$ & $12(63.2)$ & $17(56.7)$ & \multirow[t]{2}{*}{0.34} \\
\hline Right & $6(54.5)$ & $7(36.8)$ & $13(43.3)$ & \\
\hline IV tPA (n, \%) & $4(36.4)$ & $9(47.4)$ & $13(43.3)$ & 0.55 \\
\hline \multicolumn{5}{|l|}{ ASPECTS (n, \%) } \\
\hline 6 & $2(20)$ & $2(10.5)$ & $4(13.8)$ & \multirow[t]{2}{*}{0.48} \\
\hline$>6$ & $8(80)$ & $17(89.5)$ & $25(86.2)$ & \\
\hline \multicolumn{5}{|l|}{$\operatorname{CIS}(n, \%)$} \\
\hline $\operatorname{Bad}(0,1)$ & $2(20)$ & $9(47.1)$ & $10(37)$ & \multirow[t]{2}{*}{0.23} \\
\hline Good $(2,3)$ & $8(80)$ & $9(52.9)$ & $17(63)$ & \\
\hline Puncture time from symptom onset (mean, SD, min) & $195.4(111.9)$ & $224(65)$ & $213(181)$ & 0.38 \\
\hline Mean number of retrievals (n, SD) & $2.3(1.5)$ & $3.9(1.8)$ & $3.3(1.8)$ & $0.02^{*}$ \\
\hline Successful recanalization (n, \%) & $9(81.8)$ & $12(63.2)$ & $21(70)$ & 0.28 \\
\hline \multicolumn{5}{|l|}{ A. Successful recanalized ICA occlusion } \\
\hline Mean number of retrievals & $2.2(1.6)$ & $3.6(1.6)$ & $2.9(1.7)$ & 0.08 \\
\hline \multicolumn{5}{|l|}{ Number of retrievals (n, \%) } \\
\hline$<3$ & $7(77.8)$ & $2(16.7)$ & $9(42.9)$ & \multirow[t]{2}{*}{$<0.001^{*}$} \\
\hline$\geq 3$ & $2(22.2)$ & $10(83.3)$ & $12(57.1)$ & \\
\hline One time retrieval with $\mathrm{TICl} 3$ & $4(44.4)$ & $1(8.3)$ & $5(23.8)$ & $0.05^{*}$ \\
\hline \multicolumn{5}{|l|}{ Times of (mean, SD, min) } \\
\hline Total procedure time & $88(42)$ & $96(38)$ & $93(39)$ & 0.68 \\
\hline Guiding time & $27(16)$ & $15(11)$ & $20(14)$ & 0.06 \\
\hline Thrombectomy time & $61(31)$ & $80(35)$ & $72(34)$ & 0.22 \\
\hline Distal emboli (n, \%) & $0(0)$ & $9(75)$ & $9(42,9)$ & $<0.001^{*}$ \\
\hline \multicolumn{5}{|l|}{ Good Clinical outcome at 3 months (n, \%) } \\
\hline $\mathrm{mRS} \leq 2$ & $5(55.6)$ & $1(8.3)$ & $6(28.6)$ & $0.01^{*}$ \\
\hline
\end{tabular}

${ }^{*} p<0.05$ as significant. BGC : Balloon guiding catheter, SD : standard deviation, ICA: internal carotid artery, NIHSS : National Institutes of Health Stoke Scale, tPA : tissue-plasminogen activator, IA : intra-arterial, ASPECTS : Alberta Stroke Program Early CT Score, CIS : capillary index score, TICI : Thrombolysis in Cerebral Infarction, mRS: modified Rankin scale

difficulty, tortuosity of the aorta, or irritability of patients during femoral puncture. Although no difference in procedure time and thrombectomy time were observed between the BGC and non-BGC groups, it seemed to be related with our thrombectomy protocol. The Solitaire stent was deployed in the thrombus portion for $5 \mathrm{~min}$ and this protocol to allow the time to engage the thrombus into stent lumen seemed to af- fect the result.

Davalos et al. reported $85 \%$ successful revascularization and $55 \%$ good clinical outcomes (mRS $\leq 2)^{4)}$. This study reported that $74 \%$ of 141 patients used the BGC and $86 \%$ of them had anterior circulation ischemic stroke. Seventy-seven percent of 141 patients achieved good revascularization with few retrievals (mean, 1.8 retrievals), which is much lower than 
Table 5. Univariate comparison between the BGC and non-BGC group for patients with an MCA occlusion and a subgroup analysis (A) for those with successful recanalization ( $\mathrm{TICl} 2 \mathrm{~b}$ or 3 )

\begin{tabular}{|c|c|c|c|c|}
\hline Variable & $B G C(n=13)$ & Non-BGC $(n=19)$ & Total $(n=32)$ & $p$-value \\
\hline Age (SD, yrs) & $65(9)$ & $62(13)$ & $63(12)$ & 0.47 \\
\hline Women (n, \%) & $6(46.2)$ & $6(31.6)$ & $12(37.5)$ & 0.4 \\
\hline Mean initial NIHSS score (n, SD) & $17.0(7.0)$ & $17.8(3.6)$ & $17.5(5.0)$ & 0.65 \\
\hline \multicolumn{5}{|l|}{ Side } \\
\hline Left & $5(38.5)$ & $14(73.7)$ & $19(59.4)$ & $0.04^{*}$ \\
\hline Right & $8(61.5)$ & $5(26.3)$ & $13(40.6)$ & \\
\hline IV tPA (n, \%) & $8(61.5)$ & $6(31.6)$ & $14(43.8)$ & 0.09 \\
\hline \multicolumn{5}{|l|}{ ASPECTS $(n, \%)$} \\
\hline 6 & $2(20)$ & $2(10.5)$ & $4(13.8)$ & 0.48 \\
\hline$>6$ & $8(80)$ & $17(89.5)$ & $25(86.2)$ & \\
\hline \multicolumn{5}{|l|}{$\operatorname{CIS}(n, \%)$} \\
\hline $\operatorname{Bad}(0,1)$ & $4(30.8)$ & $2(11.8)$ & $6(20)$ & 0.19 \\
\hline $\operatorname{Good}(2,3)$ & $9(69.2)$ & $15(88.2)$ & $24(80)$ & \\
\hline Puncture time from symptom onset (mean, SD, min) & $195.4(111.9)$ & $224(65)$ & $213(181)$ & 0.38 \\
\hline Mean number of retrievals (n, \%) & $2.3(1.5)$ & $3.9(1.8)$ & $3.3(1.8)$ & $0.02^{*}$ \\
\hline Successful recanalization (n, \%) & $11(85)$ & $13(68)$ & $24(75)$ & 0.29 \\
\hline \multicolumn{5}{|l|}{ A. Successful recanalized MCA occlusion } \\
\hline \multicolumn{5}{|l|}{ Number of retrievals (n, SD) } \\
\hline$<3$ & $7(63.6 \%)$ & $4(30.8 \%)$ & $11(45.8)$ & 0.1 \\
\hline$\geq 3$ & $4(36.4 \%)$ & $9(69.2 \%)$ & $13(54.2 \%)$ & \\
\hline One time retrieval with $\mathrm{TICl} 3$ & $4(36.4 \%)$ & $2(15.4 \%)$ & $6(25 \%)$ & 0.23 \\
\hline \multicolumn{5}{|l|}{ Times of (mean, SD, min) } \\
\hline Total procedure time & $69(25)$ & $74(25)$ & $72(24)$ & 0.65 \\
\hline Guiding time & $16(11)$ & $21(24)$ & $19(10)$ & 0.58 \\
\hline Thrombectomy time & $53(29)$ & $53(28)$ & $53(27)$ & 0.99 \\
\hline Distal emboli (n, \%) & $5(45.5 \%)$ & $5(38.5 \%)$ & $10(41.7 \%)$ & 1 \\
\hline \multicolumn{5}{|l|}{ Good Clinical outcome (n, \%) } \\
\hline $\mathrm{mRS} \leq 2$ & $6(54.5 \%)$ & $2(15.4 \%)$ & $8(33.3 \%)$ & $0.04^{*}$ \\
\hline
\end{tabular}

${ }^{*} p<0.05$ as significant. BGC : Balloon guiding catheter, MCA: middle cerebral artery, SD : standard deviation, NIHSS : National Institutes of Health Stoke Scale, tPA: tissue-plasminogen activator, IA : intra-arterial, ASPECTS : Alberta Stroke Program Early CT Score, CIS : capillary index score, TICl : Thrombolysis in Cerebral Infarction, mRS : modified Rankin scale

other studies. The Mechanical Embolus Removal in Cerebral Ischemia (MERCI) study reported mean of 2.9 retrievals. Our study showed a mean of 2.7 retrievals (3.2 with only Solitaire stent and 2.2 with Solitaire and BGC), which was higher than that reported by Davalos et al. but lower than that of the MERCI. Our study only included anterior circulation ischemic occlusions and excluded posterior circulation ischemic occlusions for efficacy of the BGC. Mean diameter of the vertebral artery is $3.2 \mathrm{~mm}$, which is insufficient to advance a
BGC catheter (mean external diameter of distal tip, 2.55 $\mathrm{mm})^{7}$. If the BCG passes the proximal unilateral vertebral artery, contralateral flow remains in the vertebral artery. Strictly speaking, use of a BGC on posterior circulation does not induce complete flow arrest.

Our study consisted of a high proportion of ICA occlusions (48.4\%). Although our study had a 73\% successful recanalization rate, low rate (29\%) of good clinical outcomes was likely related with the proportion of occluded ICA. Because the 
Table 6. Multivariate analysis of predictors for a good clinical outcome after IA Solitaire thrombectomy for an acute anterior circulation ischemic stroke

\begin{tabular}{|c|c|c|c|}
\hline Variable & OR & $95 \% \mathrm{Cl}$ & $p$-value \\
\hline Female & 0.60 & 0.14 to 2.59 & 0.49 \\
\hline Right side of anterior circulation & 0.76 & 0.18 to 3.14 & 0.7 \\
\hline $\begin{array}{l}\text { Puncture time from symptom } \\
\text { onset (min) }\end{array}$ & 0.99 & 0.99 to 1.00 & 0.69 \\
\hline TICI 3 (total recanalized) & 0.42 & 0.09 to 1.92 & 0.26 \\
\hline Use of BGC & 5.19 & 1.07 to 25.11 & $0.04^{*}$ \\
\hline $\begin{array}{l}\text { Small retrieval numbers } \\
\text { (less than 3) }\end{array}$ & 2.94 & 0.58 to 14.74 & 0.18 \\
\hline Distal emboli & 0.49 & 0.10 to 2.25 & 0.35 \\
\hline
\end{tabular}

${ }^{*} p<0.05$ as significant. IA : intra-arterial, OR : odd ratio, $\mathrm{Cl}$ : confidence interval, $\mathrm{TICl}$ : Thrombolysis in Cerebral Infarction, BGC : balloon guiding catheter

higher proportion of ICA occlusion makes more poor outcome as other studies; the proportion of ICA occluded vessels and good clinical outcomes in each of these studies was : STAR $(18 \% \text { and } 58 \%)^{11)}$, NASA (23\% and 38\%), and Davalos et al. $(26 \% \text { and } 56 \%)^{4)}$.

BGC could induce fewer retrievals on ICA occlusion. The mean number of retrievals was 2.2 in the BGC group with successful recanalization of an ICA occlusion. Considering these data, it is significant that a BGC makes recanalization of an ICA occlusion easier, which is usually regarded as difficult. Eesa et al. also reported that manual aspiration thrombectomy through a BGC for ICA L/T occlusion was useful ${ }^{6}$. Demerath et al. emphasized the technical utility of a BGC in various cases of ICA occlusion ${ }^{5}$. Interestingly, there was no distal emboli on BGC group in a patient of successful recanalized ICA occlusion. More good clinical outcome also occurred in this group, compared with the non-BGC group in a patient of successful recanalized ICA occlusion. The BGC prevented downstream emboli during flow arrest, particularly for ICA occlusions. However, the use of BGC was not related with small retrieval numbers or the incidence of distal emboli in the successful recanalization group with MCA occlusion. The BGC could not prevent downstream of distal emboli from the MCA location effectively in our study. In other words, its effect seemed to be amplified in ICA occlusion which is near the BGC and was weaker for MCA occlusion, which is farther from the $\mathrm{BGC}$ because the manual aspiration force during flow arrest is stronger proximal than distal to the BGC.

Clot debris generated during mechanical thrombectomy and caused by many devices may contribute to clinical outcome. Distal emboli in a previously normal circulation area or blockage of collateral flow to a potentially ischemic penumbra lesion may be a contributing factor to a clinically poor outcome $^{3,9)}$. Several studies have suggested that use of a BGC during Solitaire stent thrombectomy reduced the number of distal emboli ${ }^{1,2,8)}$. Klinger-Gratz et al. reported that distal emboli were detected distal to a primary occlusion but not in adjacent territory when flow was arrest and aspiration was performed. However, in our series, two patients with an MCA occlusion had new emboli in ACA territory (one non-BGC and one BGC). Except these two patients, other distal emboli developed downstream in the same territory. Interestingly, there was no distal emboli on BGC group in a patient of successful recanalized ICA occlusion. More good clinical outcome also occurred in this group, compared with the non-BGC group in a patient of successful recanalized ICA occlusion. The BGC prevented downstream emboli during flow arrest, particularly for ICA occlusions. Preventing distal emboli during flow arrest with a BGC can lead to a good prognosis in patients with an anterior circulation ischemic stroke, particularly those with an ICA occlusion. Future devices for IA thrombectomy should consider the distal emboli because they seemed to be related with a good clinical outcome.

\section{Limitation}

This study had several limitations. First, the outcome of non-BGC patients are very poor despite successful recanalization. This could affect the good outcome in BGC group. However, overall recanalization rate were similar between two groups, and it was no significant difference. Second, an initial diffusion weighted image and digital subtraction angiography cannot differentiate between a preexisting thrombus and iatrogenic emboli downstream of the primary site of occlusion in a patients with acute stroke, so susceptibility-weighted imaging may be useful to detect the occluded thrombus and differentiate the thrombus before and after procedures to treat an anterior circulation stroke ${ }^{8}$. However, the emergency protocol at our stroke center does not include MRI for more rapid and prompt recanalization. So, distal emboli were diagnosed on angiography during IA mechanical thrombectomy. Third, left side lesion were more common in non-BGC group even 
though the NIHSS is similar. This non-symmetric distribution were appeared on subgroup analysis of MCA occlusion and it could affect the poor outcome to non-BGC group. However, both group had the similar side of lesion on ICA occlusion.

\section{CONCLUSION}

The flow arrest method using a BGC significantly reduced the number of retrievals and the occurrence of distal emboli but not procedure time. This effect was amplified in an ICA thrombus located close to the BCC tip but decreased with an MCA thrombus that was located distal to the BCG tip. The use of a BGC resulted in less frequent distal emboli and good clinical outcomes in patients with an anterior circulation ischemic stroke. We recommend use of BGC during IA mechanical thrombectomy in patients with an anterior circulation ischemic stroke, particularly those with an ICA occlusion.

\section{- Acknowledgements}

This research was supported by the Soonchunhyang University Research Fund.

\section{References}

1. Chueh JY, Kuhn AL, Puri AS, Wilson SD, Wakhloo AK, Gounis MJ : Reduction in distal emboli with proximal flow control during mechanical thrombectomy : a quantitative in vitro study. Stroke 44 : 1396-1401, 2013

2. Chueh JY, Puri AS, Wakhloo AK, Gounis MJ : Risk of distal embolization with stent retriever thrombectomy and ADAPT. J Neurointerv Surg 8 : 197-202, 2016

3. Costalat V, Lobotesis K, Machi P, Mourand I, Maldonado I, Heroum C, et al. : Prognostic factors related to clinical outcome following thrombectomy in ischemic stroke (RECOST study). 50 patients prospective study. Eur J Radiol 81 : 4075-4082, 2012

4. Davalos A, Pereira VM, Chapot R, Bonafe A, Andersson T, Gralla J, et al. : Retrospective multicenter study of Solitaire FR for revascularization in the treatment of acute ischemic stroke. Stroke 43 : 2699-2705, 2012

5. Demerath T, Reinhard M, Elsheikh S, Keuler A, Urbach H, Meckel S : Balloon guide catheter in complex anterior circulation mechanical thrombectomy : beyond proximal occlusion and flow reversal. Clin Neuroradiol 26 : 369-373, 2016

6. Eesa M, Almekhlafi MA, Mitha AP, Wong JH, Goyal M : Manual aspiration thrombectomy through balloon-tipped guide catheter for rapid clot burden reduction in endovascular therapy for ICA L/T occlusion. Neuroradiology 54 : 1261-1265, 2012

7. Ergun O, Gunes Tatar I, Birgi E, Hekimoglu B : Evaluation of vertebral artery dominance, hypoplasia and variations in the origin : angiographic study in 254 patients. Folia Morphol (Warsz) 75 : 33-37, 2016

8. Klinger-Gratz PP, Schroth G, Gralla J, Jung S, Weisstanner C, Verma RK, et al. : Protected stent retriever thrombectomy prevents iatrogenic emboli in new vascular territories. Neuroradiology 57 : 1045-1054, 2015

9. Kurre W, Vorlaender K, Aguilar-Perez M, Schmid E, Bazner H, Henkes H : Frequency and relevance of anterior cerebral artery embolism caused by mechanical thrombectomy of middle cerebral artery occlusion. AJNR Am J Neuroradiol 34 : 1606-1611, 2013

10. Nguyen TN, Malisch T, Castonguay AC, Gupta R, Sun CH, Martin CO, et al. : Balloon guide catheter improves revascularization and clinical outcomes with the Solitaire device : analysis of the North American Solitaire Acute Stroke Registry. Stroke 45 : 141-145, 2014

11. Pereira VM, Gralla J, Davalos A, Bonafe A, Castano C, Chapot R, et al. : Prospective, multicenter, single-arm study of mechanical thrombectomy using solitaire flow restoration in acute ischemic stroke. Stroke 44 : 2802-2807, 2013

12. Saver JL, Jahan R, Levy El, Jovin TG, Baxter B, Nogueira RG, et al. : Solitaire flow restoration device versus the Merci Retriever in patients with acute ischaemic stroke (SWIFT) : a randomised, parallel-group, noninferiority trial. Lancet 380 : 1241-1249, 2012

13. Tomsick T, Broderick J, Carrozella J, Khatri P, Hill M, Palesch Y, et al. : Revascularization results in the Interventional Management of Stroke II trial. AJNR Am J Neuroradiol 29 : 582-587, 2008 